Research Article

\title{
Completely Defined Co-Culture of Adipogenic Differentiated ASCs and Microvascular Endothelial Cells
}

\author{
Ann-Cathrin Volz 1,2, Larissa Hack ${ }^{1}$, Franziska B. Atzinger ${ }^{1}$ and Petra J. Kluger 1,3 \\ ${ }^{1}$ Reutlingen University, Reutlingen, Germany; ${ }^{2}$ University of Hohenheim, Stuttgart, Germany; ${ }^{3}$ Fraunhofer Institute of Interfacial Engineering and \\ Biotechnology, Department of Cell and Tissue Engineering, Stuttgart, Germany
}

\begin{abstract}
Vascularized adipose tissue models are in high demand as alternatives to animal models to elucidate the mechanisms of widespread diseases, screen for new drugs or assess drug safety levels. Animal-derived sera such as fetal bovine serum (FBS), which are commonly used in these models, are associated with ethical concerns, risk of contaminations and inconsistencies of their composition and impact on cells. In this study, we developed a serum-free, defined co-culture medium and implemented it in an adipocyte/endothelial cell (EC) co-culture model.

Human adipose-derived stem cells were differentiated under defined conditions (diffASCs) and, like human microvascular ECs (mvECs), cultured in a defined co-culture medium in mono-, indirect or direct co-culture for 14 days. The defined co-culture medium was superior when compared to mono-culture media and facilitated the functional maintenance and maturation of diffASCs including perilipin A expression, lipid accumulation, and also glycerol and leptin release. The medium also allowed mvEC maintenance, confirmed by the expression of CD31 and von Willebrand factor (vWF), and by acetylated low-density lipoprotein (acLDL) uptake. Thereby, mvECs showed strong dependence on EC-specific factors. Additionally, mvECs formed vascular structures in direct co-culture with diffASCs.

The completely defined co-culture system allows for the serum-free culture of adipocyte/EC co-cultures and thereby represents a valuable and ethically acceptable tool for the culture and study of vascularized adipose tissue models.
\end{abstract}

\section{Introduction}

Cell and tissue based in vitro models continue to gain momentum in the replacement of animal trials. But it is a major drawback that current in vitro models rely heavily on the use of animal-derived sera like fetal bovine serum (FBS). As FBS is collected from fetuses during slaughter of pregnant cows, it is considered to cause harm to the animals and is generally associated with ethical concerns (van der Valk et al., 2004). Pursuit of the 3R concept of Russel and Burch while still using such products is questionable (Russell and Burch, 1959). Also, animal sera include the risk of potential contaminations, and their constitution may vary from batch to batch (van der Valk et al., 2004; Gstraunthaler, 2003; Gstraunthaler et al., 2013). Both potential contaminations and an unstable composition causing fluctuation in product quality are considered major challenges to overcome before engineered models may be produced under Good Manufacturing Practice or Good Cell Culture Practice conform condi- tions and, e.g., be applied as human tissue implants (Hartung et al., 2002). The composition of blood serum, whether of animal or human origin, is not completely identified - neither qualitatively nor quantitatively. To avoid these drawbacks, the culture of in vitro engineered cell and tissue models has to be performed under completely defined conditions by excluding serum and all other animal-derived components, e.g., bovine brain extract. A defined medium by definition only contains factors of known structure, such as recombinant growth factors and hormones, and must exclude complex proteins or hydrolysates (van der Valk et al., 2010).

Adipose tissue (AT) has long been neglected as a target tissue in regenerative medicine and too little attention has been paid to this tissue's influence on body functions and its participation in different diseases. However, the last decades have shed light on the importance of AT's metabolic function and its relation to diseases like obesity, diabetes, pancreatitis and Morbus Crohn (Peyrin-Biroulet et al., 2007; Xu et al., 2003; Navina and Singh, 
2015). A representative model of AT is needed to further elucidate the pathways governing healthy and diseased states and to uncover further involvement of the tissue in other physiological processes. In vitro engineered tissue models have not yet reached their promised dimension of significance, which is strongly connected to the inability to engineer an adequate vascular support system. The formation of new blood vessels (vasculogenesis) and the extension of existing blood vessels (angiogenesis) are of fundamental importance for the development, enlargement or homeostasis of almost any tissue in the living body. The integration of a vascular component is especially of high importance in the engineering of AT. In vivo AT is highly vascularized, whereby each adipocyte is in contact with at least one blood vessel (Silha et al., 2005). The efficient accumulation and release of lipids and the transport of tissue-related hormones like leptin or adiponectin rely on the presence of a vascular system (Coelho et al., 2013). Additionally, the endothelial component is critical to allow constant tissue functionality and homeostasis of the tissue components (Cao, 2007, 2010). Likewise, in vitro engineered tissues rely on the integration of a vascular component. A vascular system is not only needed to scale-up and in consequence produce a sufficient volume of the tissue fragment (Yao et al., 2013a), but also for some in vitro investigations that specifically rely on the presence of a vascular component, e.g., to study the tissue's interaction with the endothelium.

The integration of a vascular supply system in AT engineering is still challenging. Current strategies to induce in vitro vascularization of AT include vascular guiding geometrics, like those present in naturally-derived decellularized matrix (Baptista et al., 2011) or the artificial biorap system (Huber et al., 2016b). Next to form-guided vascularization, de novo angiogenesis based on integrated cells is addressed in many approaches. For both, the introduction of angiogenic growth factors is feasible to support cell proliferation, migration and functionality (Nomi et al., 2006). In case de novo vascularization is sought, supportive perivascular cells, which guide endothelial cells (ECs) and support newly formed vessels, are needed (Tang et al., 2008). Various trials have addressed the vascularization of AT constructs in vitro (Aubin et al., 2015; Huttala et al., 2018; Yao et al., 2013b).

Independent of the underlying strategy, setting up a functional adipocyte/EC co-culture still represents a major milestone in the artificial vascularization of AT. It frequently has been shown that the cellular response depends on the medium ingredients, also for AT approaches (Aubin et al., 2015). Within the adjustment of the culture conditions, like cell ratio, static vs. dynamic culture, or the three-dimensional (3D) orientation of cellular components, the development of an adequate culture medium is of fundamental importance to maintain the different cell types' characteristics and functionalities and to support tissue homeostasis as a prerequisite for long-term maintenance. We have shown before that hy- drocortisone (HC) and epidermal growth factor (EGF) play a fundamental role in the co-culture of ECs and adipocytes - whether mature or developed from adipose-derived stem cells (ASCs) (Volz et al., 2017; Huber et al., 2016a). Presumably owing to the higher sensitivity of ECs compared to adipogenic differentiated ASCs (diffASCs), current approaches are in most cases based on either the addition of adipocyte factors to EC medium or on a 1:1 mixture of EC and adipocyte media (Pellegrinelli et al., 2013; Yao et al., 2013a,b; Aubin et al., 2015; Arnal-Pastor et al., 2016). Promising results, like the supportive effect of ASC co-culture on the formation of tubular structures by ECs, have been reported (Bellas et al., 2013).

The homeostasis of functional adipose tissue strongly relies on the crosstalk of the incorporated cell types (reviewed in Volz et al., 2016). Human and animal-derived sera include many different constituents (e.g., growth factors, hormones or cytokines) that may interfere with cell signaling based on soluble factors. Vascularization and the adipocytes' influence therein, e.g., rely on the factors available in the culture medium (Moya et al., 2010; Aubin et al., 2015; Yao et al., 2013b; Rajashekhar et al., 2008; Aoki et al., 2003). The existing inconsistency of the published results concerning communication between the cell types might at least partially be due to variations in the added sera. In general, the results generated in in vitro models or test systems carry a high risk of influence by unknown serum components, which may impair their reliability and reproducibility, known as the most important features of test systems in general.

The exclusion of sera or, better, all unidentified components from cell culture media is of high relevance for all tissue engineering trials. In very recent work, serum-free adipocyte/human umbilical cord vein EC co-culture was addressed, at least in some phases, with serum-free EC medium, which additionally underlines the timeliness of the topic (Huttala et al., 2018). However, so far, no completely serum-free adipocyte/EC co-culture model including a defined, custom-made co-culture medium for vascularized adipose tissue engineering has been reported. In very recent work, we successfully developed defined media for the differentiation and maintenance of adipocytes derived from ASCs (Volz and Kluger, 2018). With this study, we aimed to develop a serum-free adipocyte/EC co-culture system based on a completely defined medium composition.

\section{Material and methods}

\section{ASC isolation and expansion}

All research was carried out in accordance with the rules for investigation of human subjects as defined in the Declaration of Helsinki. As described previously, ASCs were isolated from human fatty tissue samples (Klinik Charlottenhaus, Stuttgart)

Abbreviations

3D, three-dimensional; ACLDL, acetylated low-density lipoprotein; ACM, defined adipocyte maintenance medium; ASC, adipose-derived stem cell; AT, adipose tissue CD31, cluster of differentiation 31; CoM, defined adipocyte/endothelial cell co-culture medium; DAPI, 4;6-diamidino-2-phenylindole; diffASC, adipogenic differentiated adipose-derived stem cell; EC, endothelial cell; ECBM, endothelial cell basal medium; ECM, defined endothelial cell growth medium; EGM-2mv, endothelial cell growth medium 2, microvascular; FBS, fetal bovine serum; FDA, fluorescein diacetate; MAIN, defined adipocyte maintenance mix; MSCGM, mesenchymal stem cell growth medium; MvEC, microvascular endothelial cell; PBS, phosphate buffered saline; PI, propidium iodide; RT, room temperature; VEGF, vascular endothelial cell growth factor; vWF, MvEC, microvascular
von Willebrand Factor 
of plastic surgeries received from Dr Ziegler (Volz et al., 2017; Huber et al., 2015a). Patients gave a written consent according to the permission of the Landesärztekammer Baden-Württemberg (F-2012-078; for normal skin from elective surgeries).

Initially, ASCs were seeded at a density of $5 \times 10^{3}$ cells $/ \mathrm{cm}^{2}$ in chemically defined Mesenchymal Stem Cell Growth Medium (MSCGM-CD, Lonza, Switzerland) containing 2\% fetal bovine serum (FBS) (Lonza Switzerland). Medium exchange was performed twice a week. After the first passage the medium was switched to xeno-free and serum-free MSCGM (XF/SFMSCGM, PELOBiotech, Germany) containing 5\% human platelet lysate. ASCs were used up to passage three. For the experiments, ASCs of three different donors were evaluated separately to allow for the recognition of donor-dependent effects.

\section{MvEC isolation and expansion}

Dermal microvascular ECs (mvECs) were isolated from adult human skin (Klinik Charlottenhaus, Stuttgart) as described previously (Volz et al., 2017). Briefly, human dermis was dissected into small pieces and digested in dispase solution $(2 \mathrm{U} / \mathrm{ml}$; Serva Electrophoresis, Germany) overnight at $4^{\circ} \mathrm{C}$. After the removal of the epidermis, mvECs were detached from the dermal layer by incubation with $0.05 \%$ trypsin in ethylenediaminetetraacetic acid (Life Technologies, Germany) for $40 \mathrm{~min}$ at $37^{\circ} \mathrm{C}$ and isolated mechanically into microvascular Endothelial Cell Growth Medium-2 (EGM-2mv; Lonza, Switzerland). For cell expansion, mvECs were seeded at $5 \times 10^{3}$ cells $/ \mathrm{cm}^{2}$ and grown until they reached about $90 \%$ confluence. MvECs were used up to passage three.

\section{Composition and preevaluation of co-culture media in mono-culture}

The composition of the defined adipocyte-EC co-culture medium (CoM) was developed based on defined mono-culture medium for adipocytes (adipocyte maintenance medium, ACM, developed by us (Volz and Kluger, 2018)) or ECs (EC growth medium, ECM, PELOBiotech, Germany) (Tab. 1). CoM was based on EC basal medium (ECBM), whereby additional adipocyte- and EC-specific factors, like growth factors, hormones and vitamins were added. For adipocytes a defined adipocyte maintenance mix (MAIN) was added. In all settings, EGF and HC were reduced to $5 \mathrm{ng} / \mathrm{ml}$ and $0.2 \mu \mathrm{g} / \mathrm{ml}$, respectively, as confirmed to be beneficial in our previous studies (Volz et al., 2017; Huber et al., 2016a).

To test their general suitability for either adipocytes or ECs, the media were first evaluated in mono-culture. 24-well plates were coated with $300 \mu \mathrm{l}$ of $250 \mu \mathrm{g} / \mathrm{ml}$ collagen type I (rat tail, kindly provided by the Fraunhofer IGB, Germany) solution in $0.1 \%$ acetic acid. Excess solution was aspirated and plates were dried at room temperature (RT) for about $1 \mathrm{~h}$.

ASCs were seeded in XF/SF-MSCGM at $2.5 \times 10^{4}$ cells $/ \mathrm{cm}^{2}$ in 24-well plates. After three days of expansion until confluency, adipogenic differentiation was initiated through the addition of defined adipogenic differentiation medium as described before. Differentiation was continued for 14 days with three medium exchanges per week (Fig. 1).
Tab. 1: Media composition

\begin{tabular}{|l|l|l|l|}
\cline { 2 - 4 } \multicolumn{1}{c|}{} & ACM & CoM & ECM \\
\hline Basal Medium & ABM & ECBM & ECBM \\
\hline Adipocyte Suppl. & $\mathrm{x}$ & $\mathrm{x}$ & - \\
\hline EC Suppl. & - & $\mathrm{x}$ & $\mathrm{x}$ \\
\hline
\end{tabular}

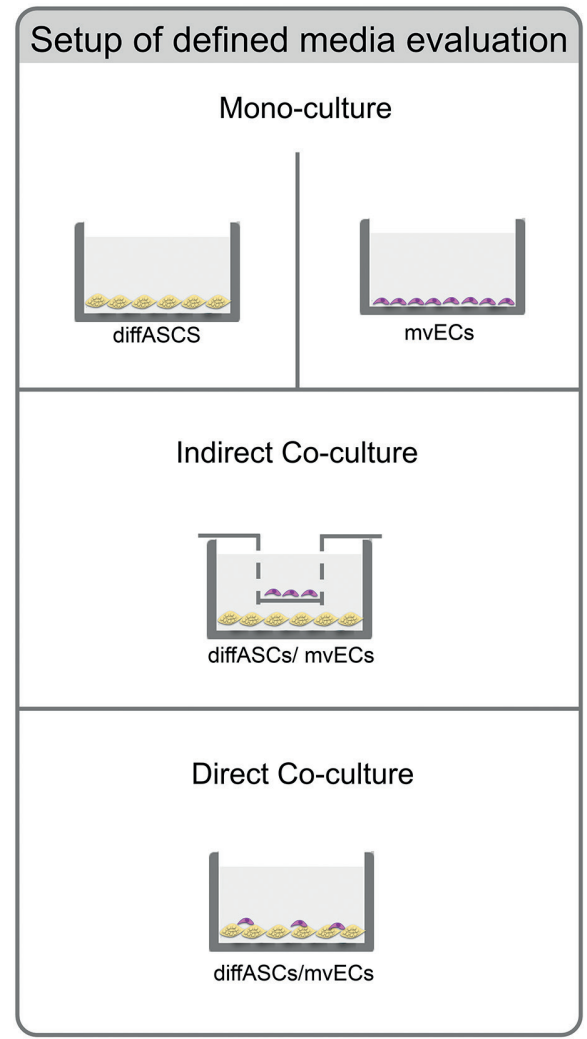

Fig. 1: Scheme of defined media evaluation in mono-culture and (in)direct co-culture

MvECs were seeded at $3.5 \times 10^{3}$ cells $/ \mathrm{cm}^{2}$ in 24-well plates and expanded for three additional days in EGM-2mv. On day 3 of mvEC expansion, respectively day 14 of adipogenic differentiation of ASCs, the medium was changed to the different test media. Cultures were continued for 14 days while the medium exchange took place twice per week.

Evaluation of co-culture media in (in)direct diffASC/EC co-culture For indirect co-culture, ASCs were seeded and differentiated analogously to the mono-culture. On day 13 of differentiation, $1.3 \times 10^{3}$ cells $/ \mathrm{cm}^{2} \mathrm{mvECs}$ of three different donors were seed- 
ed separately in collagen-coated inserts of $0.4 \mu \mathrm{m}$ pore size in EGM-2mv. After $24 \mathrm{~h}$ of cell attachment, inserts were transferred into the well plates with diffASCs and cultured in the different defined culture media for 14 further days.

For direct co-culture, $8 \times 10^{3}$ cells $/ \mathrm{cm}^{2}$ mvECs were directly seeded on top of diffASCs in 24-well plates and cultured in CoM for 14 days.

Parallel to the different co-culture settings, diffASCs and mvECs were cultured in mono-culture in their specific media, i.e., ACM or ECM, respectively, as controls. Medium was changed twice per week.

\section{Viability evaluation}

Cell viability in the different media was evaluated based on a live/dead staining on day 0,7 , and 14. Cells were washed in phosphate buffered saline (PBS, Biochrom, Germany) before addition of the staining solution, consisting of $10 \mu \mathrm{g} / \mathrm{ml}$ fluorescein diacetate (FDA, Sigma Aldrich, Germany) and $5 \mathrm{mg} / \mathrm{ml}$ propidium iodide (PI, Sigma Aldrich, Germany) in Dulbecco's Modified Eagle Medium (Biochrom, Germany), for $15 \mathrm{~min}$ at $37^{\circ} \mathrm{C}$. Cells were imaged in PBS at RT with an Axio Observer microscope (10x objective) and an Axiocam 506 mono using ZEN software (all Zeiss, Germany).

\section{Oil-red-O staining}

On day 0,7 , and 14 an Oil-Red-O staining was performed to evaluate the level of accumulated lipids in the diffASCs. The staining was evaluated quantitatively and qualitatively as described previously (Volz et al., 2017). Images were taken with an Axiovert 135 microscope (10x objective) and an Axiocam 105 color using ZEN software (Zeiss, Germany).

\section{Immunofluorescence staining}

Perilipin A, a membrane protein located on adipocyte lipid vacuoles, as well as CD31 and von Willebrand factor (vWF), both endothelial proteins, were evaluated as markers of cell specificity on day 0,7 , and 14 by immunofluorescence staining. Cells were fixed in 4\% paraformaldehyde for $10 \mathrm{~min}$. For the intracellular markers $\mathrm{vWF}$ and perilipin A, cells were permeabilized for 10 min with $0.1 \%$ Triton X (Sigma, Germany). Cells were then incubated in blocking solution, consisting of $3 \%$ bovine serum albumin (Biomol, Germany) in $0.1 \%$ Triton $\mathrm{X}$ for $30 \mathrm{~min}$ to block unspecific binding sites. The primary antibody against perilipin A was rabbit-derived (P1998, lot: 09544801V, Sigma Aldrich, Germany) and applied in the ratio 1:300. The antibodies against CD31 (M082301-2, lot: 20045564, Dako, Germany) and vWF (sc53466, lot: B0117, Santa Cruz, Germany) were both derived from mouse and applied in the ratio 1:50. All antibodies were diluted in blocking solution and incubated for $3 \mathrm{~h}$ at RT. For perilipin A, a goat-derived Alexa Fluor ${ }^{\mathrm{TM}}$ 488-conjugated secondary antibody (ab150077, lot: GR322463, Abcam, GB) was applied 1:250 in blocking solution for $30 \mathrm{~min}$ at RT, while an anti-mouse Cy3-conjugated antibody from goat (115-165-003, lot: 118666, Dianova, Germany) was incubated 1:250 and used to visualize CD31 and vWF. Cell nuclei were counterstained with $1 \mu \mathrm{g} / \mathrm{ml}$ 4',6-diamidino-2-phenylindole (DAPI, Serva Electrophoresis,
Germany) in PBS for 15 min. Finally, cells were imaged in PBS at RT using a 10x objective. Z-stack images were taken with an Axio Observer microscope and an Axiocam 506 mono using ZEN software (Zeiss, Germany) and combined using the function "extended depth of focus".

\section{Quantitative analysis of vascular-like structures}

Vascular-like structures were analyzed based on the immunofluorescence images of the antibody staining against CD31 with Image J. Total vessel length, number of structures, and number of nodes per $1 \mathrm{~mm}$ structure were evaluated.

\section{Leptin ELISA}

Leptin release was measured using an ELISA Kit (Pepro Tech, Germany) according to the manufacturer's protocol based on cells' $24 \mathrm{~h}$ culture supernatants in a 1:1 dilution. $100 \mu \mathrm{l}$ 3,3',5,5'-tetramethylbenzidine substrate (Sigma-Aldrich, Germany) were added to each well for approx. $20 \mathrm{~min}$ at RT for color development. The wells were read out at $650 \mathrm{~nm}$ with a wavelength correction set at $470 \mathrm{~nm}$ (Omega Fluostar; BMG Labtech, Germany).

\section{Glycerol assay}

To determine the adipocytes' lipolytic rate, glycerol release was evaluated based on cells' $24 \mathrm{~h}$ supernatants with a glycerol quantification kit (Randox, Ireland). Briefly, $55 \mu 1$ samples were used in a 1:5 dilution, mixed with $100 \mu \mathrm{l}$ reagent and incubated for $20 \mathrm{~min}$ at RT in duplicates. Color development was read out at 520 nm (Omega Fluostar; BMG Labtech, Germany).

\section{Acetylated low-density lipoprotein intake}

By using the scavenger pathway, mvECs are able to take in acetylated low-density lipoprotein (acLDL). To test this EC-functionality, Alexa Fluor ${ }^{\mathrm{TM}}$ 488-labeled acLDL (Invitrogen, Germany) was mixed 1:1000 in EGM-2 and cells were incubated for $4 \mathrm{~h}$ at $37^{\circ} \mathrm{C}$. After a rinsing step with PBS, nuclei were counterstained with $0.1 \mu \mathrm{g} / \mathrm{ml}$ Hoechst 33342 (Sigma Aldrich, Germany) for 15 min at $37^{\circ} \mathrm{C}$. Finally, cells were imaged in PBS at RT with an Axio Observer microscope (10x objective) and an Axiocam 506 mono using the software ZEN (all Zeiss, Germany).

\section{Statistics}

All experiments were performed repeatedly, using cells from at least three different biological donors. Outliers were identified by a Grubbs' test and excluded from the data set. The obtained data was compared by a one-way analysis of variance (ANOVA) with repetitive measurement and a Tukey post-hoc test using OriginPro 2017. Vascularization was evaluated with a Wilcoxon test. Statistic significance was stated as $*, p<0.05 ; * *, p<0.01$ and $* * *, \mathrm{p}<0.001$.

\section{Results}

The development of vascularized AT equivalents to replace soft tissue and to generate in vitro test systems is still a major challenge. A vascular network is indispensable for the suffi- 
cient supply of a construct. The prerequisite for successful establishment of the model is the definition of suitable culture conditions. In this study, we evaluated the suitability of a defined co-culture medium for an adipocyte/EC co-culture model with regard to cell maintenance in diffASC and mvEC mono-cultures, as well as in an indirect and direct co-culture model of both cell types.

\subsection{Defined co-culture medium facilitates functional diffASC mono-culture}

To test and compare their general suitability, diffASCs were cultured in ACM, CoM or ECM for 14 days. The media's influence on cell viability was evaluated with a live/dead staining, and an immunofluorescence staining against perilipin A was performed to test for adipocyte-specific characteristics. Accumulated lipids were visualized and analyzed quantitatively via an Oil-red-O staining (Fig. 2).

Although diffASC viability was high in all media, viability was higher in ECBM-based media (CoM and ECM) compared to ACM (Fig. 2A-C). Many perilipin A-positive cells could be found upon culture in all media; however, some non-expressing cells were also present in each case, indicating that not all of the cells committed to the adipogenic lineage or that some of the cells dedifferentiated during the maintenance phase (Fig. 2D-F). The level of perilipin A expression was lowest in ACM. Additionally, in contrast to the ECBM-based media, some of the diffASCs in ACM still exhibited an elongated, fibroblast-like morphology and incorporated fewer, smaller lipid vacuoles compared to cells in the other test media (Fig. 2G-I).

Lipid storage of diffASCs after 14 days of differentiation (day 0 ) was set as $100 \%$. The quantitative analysis demonstrated an increase in incorporated lipids in all media after 7 and 14 days with the lowest content observed in ACM with $178 \%( \pm 31.5)$ and $230 \%$ ( \pm 75.6$)$ (Fig. 2J). The results were comparable between culture in ECM $(198 \%( \pm 27.2)$ and $271 \%( \pm 72.4))$ and CoM $(208 \%( \pm 39.6)$ and $320 \%( \pm 84.4))$. Lipid storage of diffASCs in CoM was significantly higher compared to all other media and timepoints except for ECM on day 14, indicating a better adipogenic development in the composed co-culture medium (overview of detailed data in Tab. $\mathrm{S}^{1}{ }^{1}$ ).
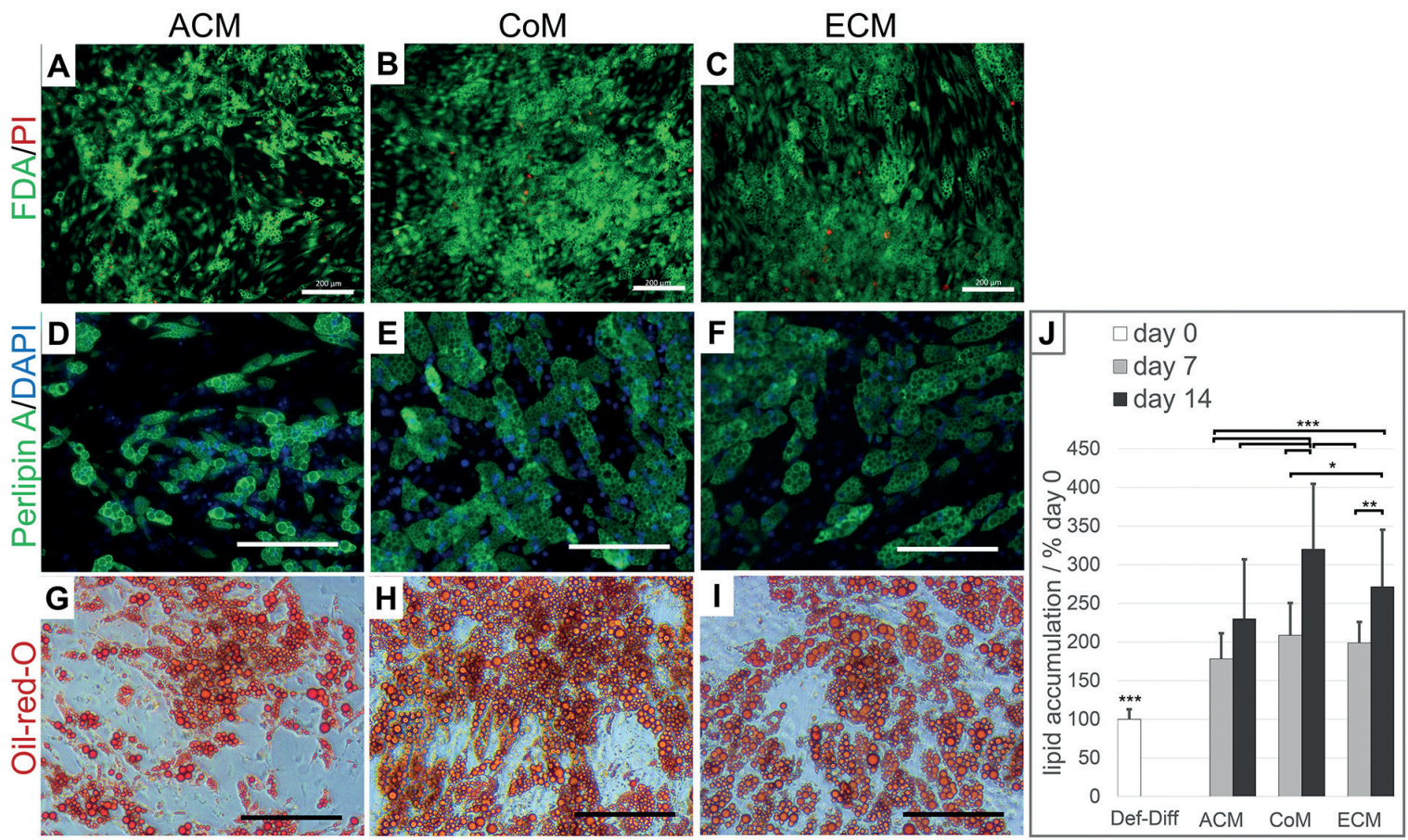

Fig. 2: DiffASC mono-culture on day 14

$2.5 \times 10^{4}$ cells $/ \mathrm{cm}^{2}$ ASCs were seeded in XF/SF-MSCGM in collagen type I coated 24-well plates. After three days, medium was change to the different test media ACM, CoM or ECM and culture was continued for 14 days with three medium exchanges per week. (A-C) FDA/ $\mathrm{PI}$ - viability staining: living cells are shown in green, dead cells in red. (D-F) Perilipin A/DAPI staining: specific marker expression is shown in green, nuclei are stained in blue. (G-I) Oil-red-O staining: lipids were stained with Oil-Red-O, visible in red. (J) Quantitative analysis of lipid accumulation of diffASCs on day 7 and day 14 in mono-culture; lipid storage of diffASCs immediately after differentiation (day 0 ) was set as $100 \%$. *, $\mathrm{p}<0.05 ;{ }^{* *}, \mathrm{p}<0.01 ;{ }^{* * *}, \mathrm{p}<0.001$; scale bar: $200 \mu \mathrm{m}$. Data were evaluated from three independent donors in duplicates. Quantitative data are displayed as means. Representative images were selected from one donor.

1 doi:10.14573/altex.1802191s 


\subsection{Defined co-culture medium facilitates functional mvEC mono-culture}

To evaluate cell morphology and prove the expression of EC-specific markers, CD31 and vWF were evaluated next to a live/dead stain for mvECs on day 14 in the different test media (Fig. 3). In the mono-cultures of mvECs, ACM did not support cell attachment. In contrast, visible mvECs were viable on day 14 in both CoM and ECM and built a confluent monolayer with EC-specific cobblestone-like morphology as well as vWF and CD31 expression. The acLDL uptake of mvECs was analyzed to evaluate EC functionality. After 14 days of maintenance, mvECs in all media but ACM retained their ability to take up acLDL.

\subsection{Defined co-culture medium supports indirect diffASC/mvEC co-culture}

An indirect co-culture study was performed over 14 days to test diffASC and mvEC behavior in the media in a more physiological co-culture and to elucidate a possible influence of the cell types on each other through soluble factors.

As seen in the mono-culture, the immunofluorescence and the Oil-red-O staining demonstrated a higher perilipin A expression, a higher amount of lipid vacuoles and a more roundish cell morphology in the ECBM-based media compared to ACM (Fig. 4G). In all approaches, the diffASCs showed an increase in lipid accumulation between day 0 and day 7 but then, in contrast to the mono-culture (see Section 3.2), reduced their lipid levels non-significantly until day 14 (Fig. 4G, Tab. S2 ${ }^{1}$ ). The lowest lipid accumulation was seen in ACM mono-culture $(197 \%( \pm 103.9)$ and $130 \%( \pm 27.3))$.

To determine the functionality and lipolytic rate of diffASCs, the release of leptin and glycerol were measured in the cell supernatants on day 7 and 14. The levels immediately after differentiation were set as 100\%, respectively (Fig. 4G-I). On day 7, leptin release of diffASCs was significantly higher compared to day 0 in all test media. This supports the overall hypothesis that diffASCs further gain adipocyte-specific functions during the culture period. Comparable levels of leptin release were reached in ACM and ECM (Fig. 4H, Tab. S2 ${ }^{1}$ ). The highest levels were detected in CoM with $3766 \%( \pm 2642.3)$ and $2195 \%( \pm 1579.9)$, and the lowest in ACM mono-culture with $858 \%( \pm 622.7)$ and $76 \%( \pm 124.9)$. In general, on day 14 , the leptin release was lower than on day 7 in all media but had not returned to baseline, which is consistent with the decreasing lipid content between day 7 and day 14. CoM supported the highest levels of lipid accumulation

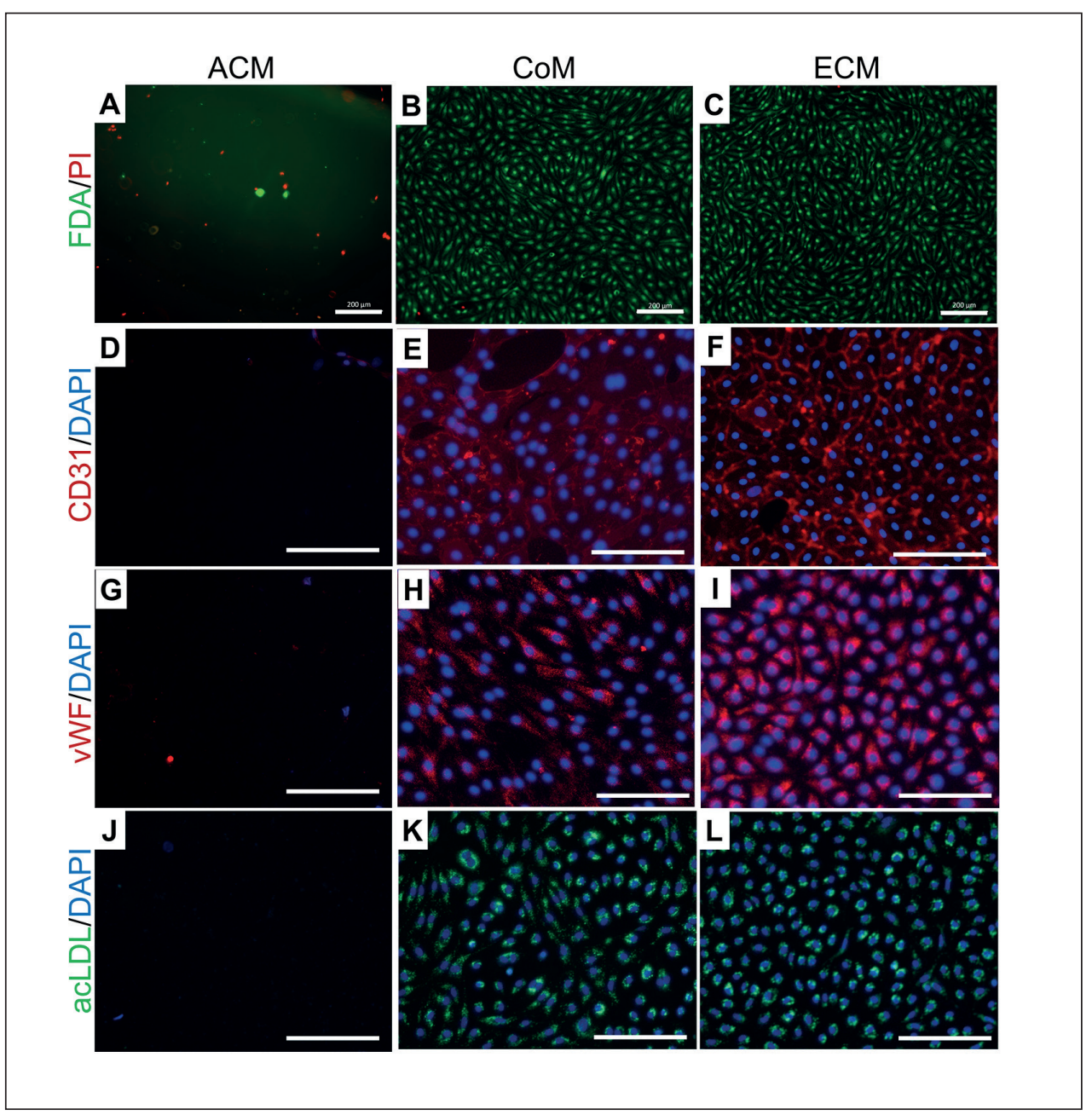

Fig. 3: MvEC monoculture on day 14

$3.5 \times 10^{3}$ cells $/ \mathrm{cm}^{2}$ mvECs were seeded in collagen I coated 24-well plates and expanded for three days in EGM-2mv. Then, the medium was changed to the different test media and cultures were continued for 14 days. Medium was exchanged twice per week. (A-C) FDA/ $\mathrm{PI}$ - viability staining: living cells are shown in green, dead cells in red. (D-F) CD31/DAPI staining: CD31 expression is shown in red, nuclei are stained in blue. (G-I) VWF/DAPI staining: vWF expression is shown in red, nuclei are stained in blue. (J-L) AcLDL assay: acLDL incorporated in cells is shown in green, nuclei were stained in blue, scale bar: $200 \mu \mathrm{m}$. Data were evaluated from three independent donors in duplicates. Representative images were selected from one donor. 


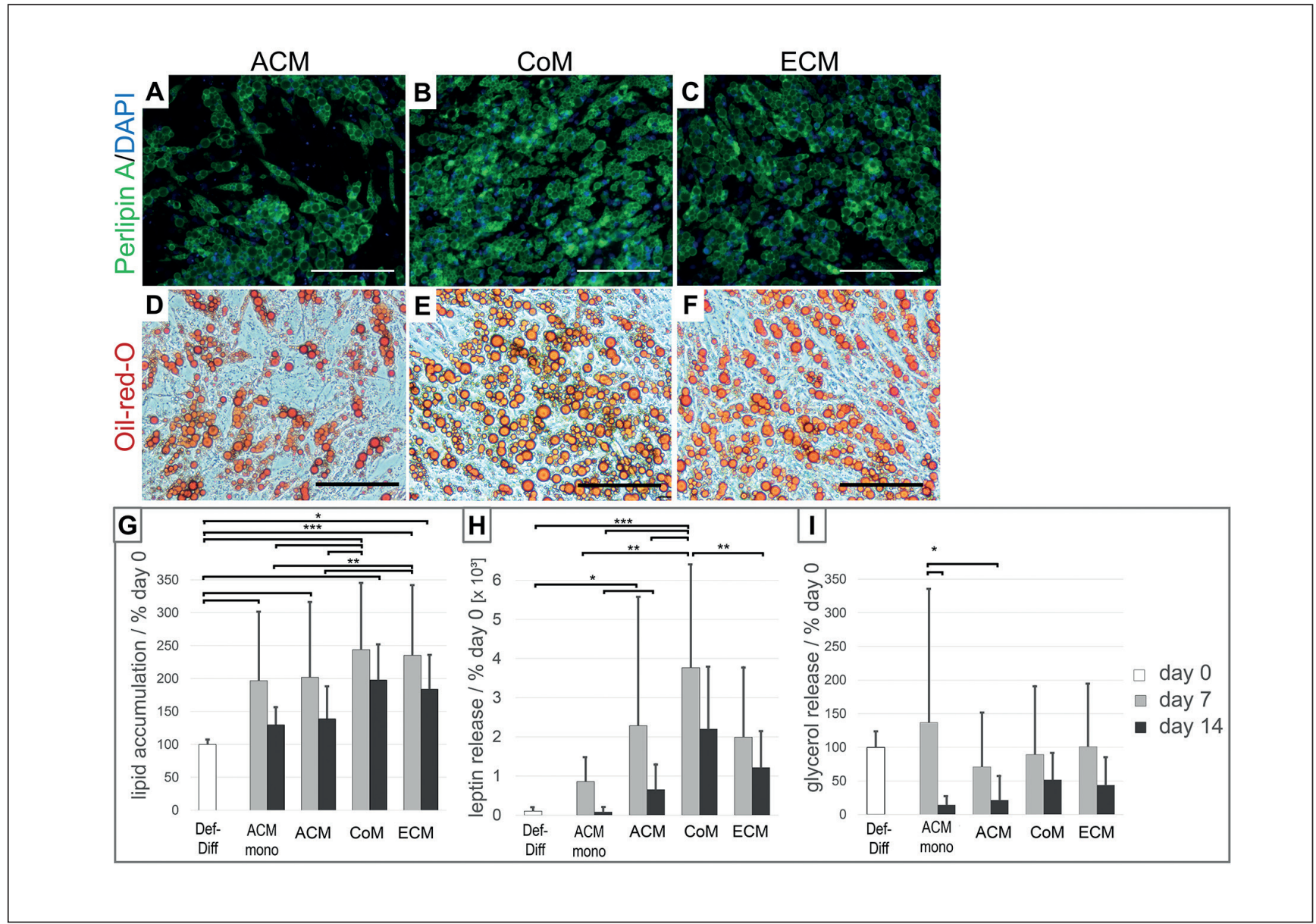

Fig. 4: DiffASCs in indirect co-culture with mvECs on day 14

ASCs were seeded and differentiated analogously to the mono-culture. On day 13 of ASC differentiation, mvECs of three different donors were seeded separately in collagen-coated inserts in EGM-2mv. After $24 \mathrm{~h}$, inserts were transferred into the well plates with diffASCs and cultured in the different defined culture media ACM, CoM and ECM for 14 further days.

(A-C) Perilipin A/DAPI staining: marker expression is shown in green, nuclei are stained in blue. (D-F) Oil-red-O staining: lipids were stained with Oil-Red-O and are visible in red. (G) Quantitative analysis of lipid accumulation. (H) Quantitative analysis of leptin release of diffASCs. (I) Quantitative analysis of glycerol release; values of diffASCs immediately after differentiation (day 0 ) were set as $100 \%$. *, $\mathrm{p}<0.05 ;{ }^{* *}, \mathrm{p}<0.01 ;{ }^{* * *}, \mathrm{p}<0.001$; scale bar: $200 \mu \mathrm{m}$. Data were evaluated from three independent donors in duplicates. Quantitative data are displayed as means. Representative images were selected from one donor.

and leptin release in all approaches, highlighting its superior influence on adipocyte development. This is especially visible on day 7, when CoM led to significantly higher leptin values compared to the other media. While lipid accumulation in ACM was independent of the presence of ECs, leptin release was increased in the presence of mvECs, indicating a higher diffASC functionality.

Glycerol release was comparable in ACM, in CoM and ECM (Fig. 4I, S2 ${ }^{1}$ ). In general, glycerol levels decreased between day 7 and day 14 in all approaches (significantly for ACM in mono-culture with $14 \%( \pm 12.6)$ on day 14 , which was preceded by a remarkable rise to $137 \%( \pm 198.8)$ on day 7$)$.

After 14 days in indirect co-culture with diffASCs, mvECs in CoM and ECM again orientated themselves in even monolayers, expressed CD31 and vWF throughout and were able to take up acLDL (Fig. 5). In line with the results displayed in section 3.2, mvECs in ACM showed poor cell attachment in ACM and were unable to build an even monolayer. However, in contrast to mvECs mono-cultured in ACM, some cells were still adherent on day 14 and showed some extent of marker expression, even if no functional acLDL uptake was detectable.

\subsection{Direct co-culture supports the maintenance of diffASCs and mvECs and leads to the formation of vascular-like structures in defined conditions}

DiffASCs and mvECs were co-cultured in CoM for 14 days to evaluate possible benefits of direct cell-cell interaction (Fig. 6).

After two weeks in direct co-culture, most diffASCs still exhibited perilipin A expression and accumulated lipids. However, some cells lacking associated adipocyte markers were observed. 

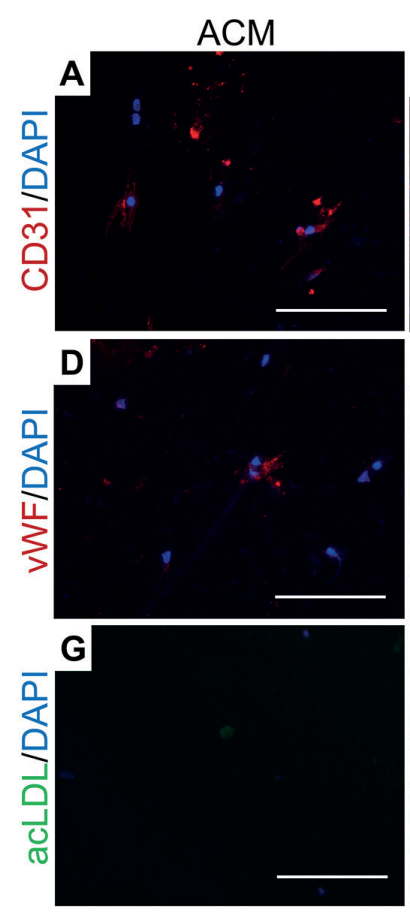
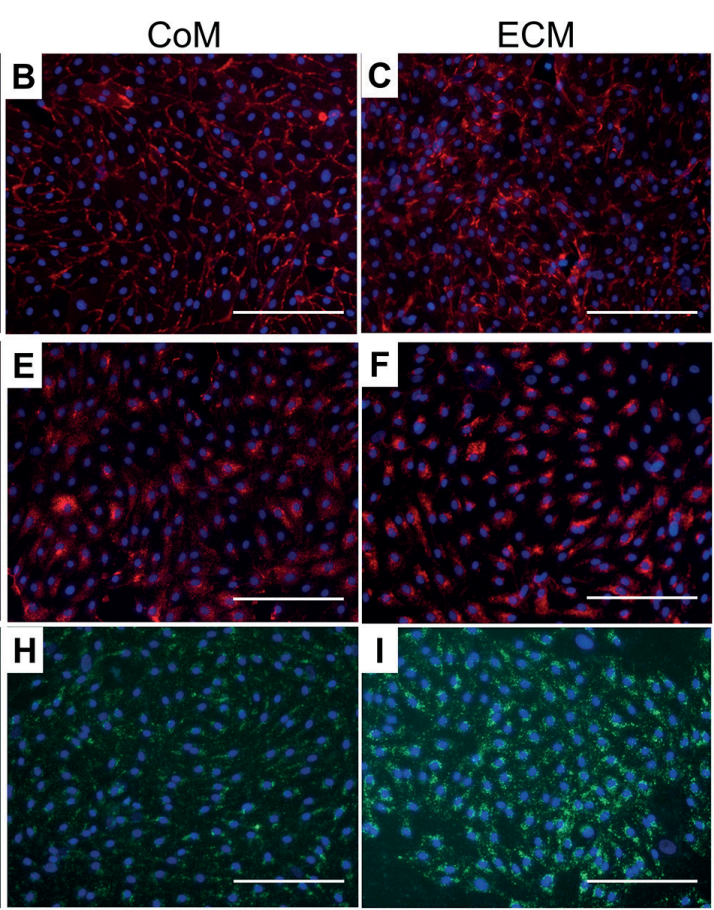

In both the mono- and the co-culture, the levels of accumulated lipids increased significantly to $280 \%( \pm 98.7)$ and $277 \%( \pm 93.6)$ on day 7 , respectively. On day 14 , only a small, non-significant decrease was recorded (Fig. 6A, Tab. S3 ${ }^{1}$ ).

In mono- and co-culture, the leptin values increased greatly on day 7 , compared to day $0\left(\mathrm{Tab} . \mathrm{S}^{1}\right)$. Levels on day 14 were comparable, indicating similar functionalities within the different approaches (Fig. 6 B, Tab. S3 ${ }^{1}$ ).

Glycerol release increased to $148 \%( \pm 47.5)$ on day 7 and to $236 \%( \pm 79.1)$ on day 14 in mono-culture. In the co-culture, glycerol increased to $229 \%( \pm 78.1)$ on day 7 and stayed at $229 \%$ $( \pm 89.3)$ on day 14 (Fig. 6C, Tab. S3 ${ }^{1}$ ). Thus, glycerol release was increased in all approaches; only the mono-culture at day 7 showed relatively low levels of released glycerol.

MvECs exhibited a strong expression of CD31. Remarkably, mvECs formed vessel-like structures, which were organized in a vascular-like network in-between the diffASCs. Such structures were not observed in the mono-culture of mvECs of the same donors (Fig. 6I). The vascular structures in the direct co-culture were evaluated quantitatively in terms of the total structure length per area, the number of structures per area and existing nodes within the structures. Structure lengths in co-culture showed absolute values of $4.9( \pm 7.24) \mathrm{mm} / \mathrm{cm}^{2}$. When values were normalized to $100 \%$ within the donors, they showed a standard deviation of $\pm 31.8 \%$. The amount of structures was $7.6( \pm 7.16) / \mathrm{cm}^{2}$ on average, respectively $100 \%( \pm 32.5)$. Within the structures, nodes were detected with a frequency of $1.1( \pm 2.57)$ nodes $/ \mathrm{mm}$ structure. At higher magnifications (Fig. $6 \mathrm{H}$ ), close proximity of mvECs with diffASCs and with undifferentiated ASCs is visible as stained nuclei with and without surrounding perilipin A expression.
Fig. 5: MvECs in indirect coculture with diffASCs on day 14 ASCs were seeded and differentiated analogously to the mono-culture. On day 13 of ASC differentiation, mvECs of three different donors were seeded separately in collagen-coated inserts in EGM-2mv. After $24 \mathrm{~h}$, inserts were transferred into the well plates with diffASCs and cultured in the different defined culture media ACM, CoM and ECM for 14 further days. (A-C) CD31/DAPI staining: CD31 expression is shown in red, nuclei are stained in blue. (D-F) VWF/DAPI staining: vWF expression is shown in red, nuclei are stained in blue. (G-I) AcLDL assay: acLDL incorporated in cells is shown in green, nuclei were stained in blue, scale bar: $200 \mu \mathrm{m}$. Data were evaluated from three independent donors in duplicates. Representative images were selected from one donor.

Some cells with stored acLDL were visible in the direct co-culture; however, their orientation suggests that these cells did not match with the cells of the vascular-like structures in all cases.

\section{4}

\section{Discussion}

Previous results, like the establishment of a functional, vascularized adipose tissue construct by Sorrell et al. (2011), showed great progress towards the application of tissue engineered constructs. In this study, we aimed to develop a defined co-culture medium, which has the potential to benefit many existing approaches to vascularized adipose tissue engineering without the ethical and safety concerns associated with the use of FBS.

Within the analysis of the general suitability of the media, ECBM-based media showed a superior effect on diffASC survival and differentiation compared to the adipocyte-specific medium ACM. CoM resulted in the best adipocyte maturation. ACM and CoM both contain the adipocyte-specific factors (MAIN) but resulted in significantly different levels of lipid accumulation. According to this, it can be concluded that ECM is supplemented adequately to fulfill the requirements of both mvECs and diffASCs. It appeared that diffASCs are not completely dependent on the classical adipogenic factors in ACM as long as the basal medium is well composed and they are supplied with a variety of growth factors. However, the factors and nutrients in ECM and the AC-specific factors synergistically support adipocyte maintenance.

A functional EC culture is, amongst others, characterized by the expression of EC-specific markers like vWF and CD31, the ability 

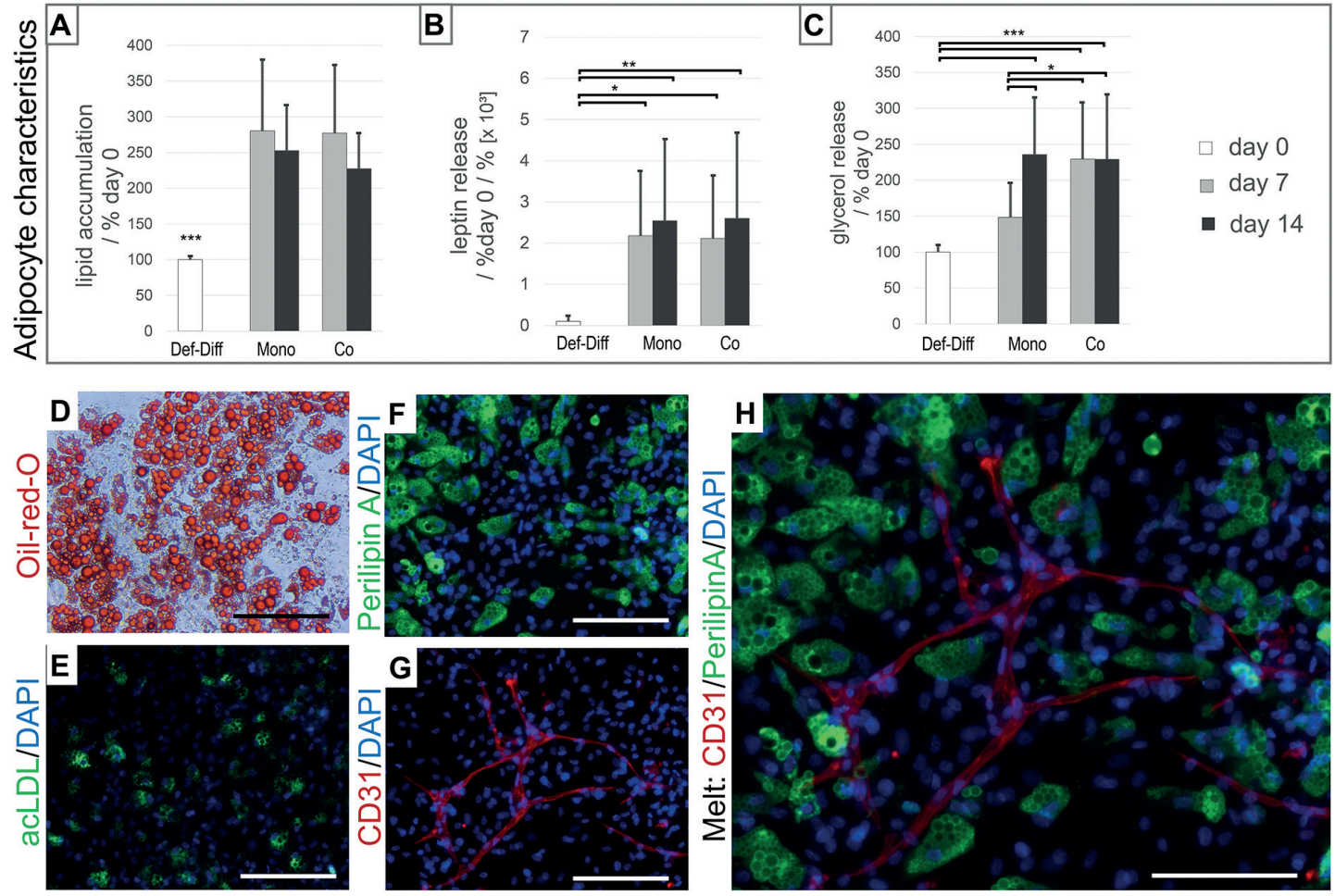

\begin{tabular}{|c|c|c|c|c|c|}
\hline I & Units & Co & Mono & $\mathrm{p}$-value & Significance level \\
\hline \multirow{2}{*}{$\begin{array}{c}\text { Total structure length } \\
\text { per area }\end{array}$} & [\%] & $100 \pm 31.8$ & $0 \pm 0.0$ & $1.95 \times 10^{-3}$ & ** \\
\hline & {$\left[\mathrm{mm} / \mathrm{cm}^{2}\right]$} & $4.9 \pm 7.24$ & $0.0 \pm 0.00$ & n.i. & n.i. \\
\hline \multirow{2}{*}{ Structures per area } & {$[\%]$} & $100 \pm 32.5$ & $0 \pm 0.0$ & $4.88 \times 10^{-4}$ & $* * *$ \\
\hline & {$\left[1 / \mathrm{cm}^{2}\right]$} & $7.6 \pm 7.16$ & $0.0 \pm 0.00$ & n.i. & n.i. \\
\hline $\begin{array}{l}\text { Nodes per structure } \\
\text { length }\end{array}$ & {$[1 / \mathrm{mm}]$} & $1.1 \pm 2.57$ & n.d. & n.d. & n.d. \\
\hline
\end{tabular}

Fig. 6: DiffASCs and mvECs in direct co-culture on day 14

For direct co-culture, $8 \times 10^{3}$ cells/cm² mvECs were directly seeded on top of diffASCs in 24-well plates and cultured in CoM for 14 days. (A) Quantitative analysis of lipid accumulation. (B) Quantitative analysis of leptin release of diffASCs. (C) Quantitative analysis of glycerol release; values of diffASCs immediately after differentiation (day 0) were set as $100 \%$. (D) Oil-red-O staining: lipids were stained with Oil-red-O and are visible in red. (E) AcLDL assay: acLDL incorporated in cells is shown in green, nuclei were stained in blue. (F-H) Perilipin A/CD31/DAPI staining: specific marker expression is shown in green and red, nuclei were stained in blue. (I) Quantitative analysis of vascularization on day 14; values of vascular-like structures on day 14 in co-culture were set as $100 \%,{ }^{*}, p<0.05$; ${ }^{* *}, p<0.01$; ${ }^{* \star *}, \mathrm{p}<0.001$; scale bar: $200 \mu \mathrm{m}, \mathrm{n} . \mathrm{i} .=$ not investigated, n.d. $=$ not detectable. Data were evaluated from three independent donors in duplicates. Quantitative data are displayed as means. Representative images were selected from one donor.

to take up acLDL and the formation of a dense monolayer, including tight cell-cell contacts. Especially the latter represents an important feature to maintain EC barrier function in vivo (Bazzoni and Dejana, 2004). The adipogenic supplements, added in ACM and CoM, did not have a clear effect on the behavior of mvECs. However, ACM alone did not support a functional EC culture, seen by almost complete cell detachment on day 14 . This clearly highlights a strong reliance of mvECs on the presence of EC factors like vascular endothelial growth factor (VEGF) and insulin-like growth factor, which we already confirmed previously in studies based on serum-containing media (Volz et al., 2017; Huber et al., 2016a). We conclude, based on the mono-culture evaluation, that CoM and ECM both represent suitable serum-free, defined media for a diffASC/mvEC co-culture, whereby CoM lies ahead somewhat with regard to diffASC culture.

To test for a paracrine influence of the cell types on each other in the different test media, diffASCs and mvECs were co-cultured in an indirect setting for 14 days post differentiation. In the main, 
the results obtained in the mono-culture were reproduced here. Lipid accumulation, perilipin A expression and leptin release were again higher in CoM and ECM compared to ACM in the indirect co-culture setting, which confirms these two as the most suitable media with regard to adipocyte development. The direct comparison of mono- and co-cultured diffASCs did not reveal any differences in lipid accumulation and no clear stimulation of adipogenic differentiation by the presence of mvECs, as shown by others and ourselves previously (Yao et al., 2013a; Fukumura et al., 2003; Volz et al., 2017). However, others substantiated an anti-adipogenic effect of ECs in crosstalk (Rajashekhar et al., 2008). Ultimately, the ECs' role in adipocyte development appears to depend on other conditions. The significantly increased leptin levels in ACM co-culture compared to the mono-culture suggest a supportive role of mvEC signaling on diffASC functionality. This hypothesis is supported by the significantly elevated glycerol levels in ACM mono-culture on day 7, which indicate an early induction of lipolysis, possibly induced by dedifferentiation (Marcinkiewicz et al., 2006; Huber and Kluger, 2015). In line, the very low glycerol levels in ACM mono-culture on day 14 might be explained by the low remaining lipid levels in the culture due to early dedifferentiation in the absence of ECs.

In contrast to the mono-culture, lipid contents decreased between day 7 and 14 in the co-culture and the mono-culture control. This difference might be explained by the different medium volumes, which were three times greater in the indirect co-culture compared to the mono-culture. Adipogenic differentiation and lipogenesis are known to depend on autocrine stimulation, e.g., with angiopoietin II, insulin-like growth factor, nitric oxide, VEGF or adiponectin (reviewed in Huber et al., 2015b; Volz et al., 2016), which might have been diluted by the higher medium volume. However, the incorporated lipids and leptin stayed at levels clearly above those of day 0 , while glycerol decreased to values below the baseline in all settings on day 14, indicating a low lipolytic state of the adipocytes in general (Galic et al., 2010). Therefore, the results show a well-developed cell functionality, especially in CoM, and support the hypothesis of a self-equilibrating state of homeostasis that develops over the 14 days of culture.

In contrast to the mono-culture setting in ACM, some mvECs were still preserved and adherent in the co-culture setting on day 14. The cells exhibited EC-specific marker expression of CD31 and vWF. DiffASCs therefore potentially produced some pro-endothelial or pro-angiogenic factors. Feasible factors could be angiopoietin II, basic fibroblast growth factor, hepatocyte growth factor, VEGF and leptin (Volz et al., 2016). However, the levels were not sufficient to compensate for the absence of endothelial-specific factors, which were only available in ECM and CoM, in which mvECs showed adequate maintenance. To sum up, CoM is also suitable for the indirect co-culture of diffASCs and mvECs. The crosstalk therein will be part of future investigations. Furthermore, a possible support of diffASCs by soluble mvEC factors in the adipocyte factor-free ECM must be examined.

CoM also proved to be suitable for the direct co-culture of diffASCs and mvECs. DiffASCs maintained and further increased their accumulated lipids as well as their perilipin A expression and leptin release, while mvECs retained their cell-specific marker expression. Additionally, the conditions including the culture medium facilitated and supported the formation of vascular-like structures in the presence of diffASCs.

Glycerol and lipid levels uniformly increased after day 0 in all replicates and confirm the further development of the diffASCs. The elevated glycerol levels are in accordance with early findings correlating lipid stores with glycerol release (Jacobsson and Smith, 1972) and suggest an increase in the basal lipolytic rate. Leptin again showed a disproportional elevation to high levels, 20 times above the values detected on day 0 . The very high leptin levels thereby indicate a fundamental increase in the cells' functionality. The absence of noticeable differences between the mono- and co-culture do not suggest a possible beneficial influence of mvECs on diffASCs differentiation. Both a pro-differentiating (Yao et al., 2013a; Aubin et al., 2015) and a triggered dedifferentiating effect of ECs on adipocytes has been reported by other groups (Hutley et al., 2001; Rajashekhar et al., 2008; Aoki et al., 2003; Moya et al., 2010). Further investigations are necessary to evaluate the endothelial-derived effects (beneficial or adverse) on a more molecular level. Further optimizations of the model might allow intensification of the ECs' effect and permit its detection more easily. The maintenance of not only lipid and glycerol but also leptin levels might be due to a homeostatic state of the culture, which was adjusted in a self-organized manner between day 0 and 7 and maintained until day 14 .

In direct co-culture, cells with a noticeable acLDL uptake were present. The assignment of the functionality to mvECs was however difficult. We detected a noticeable acLDL incorporation in mono-cultures of diffASCs in CoM in another setup (data not shown). This functionality has been reported for ASCs before, particularly after ASC culture in EC media or as a response to the application of shear stress, but to some extent as well in the undifferentiated state (Ikegame et al., 2011; Chen et al., 2014). In the studies mentioned, this function has been attributed to the endothelial differentiation of ASCs. So far, acLDL uptake has neither been associated with adipogenic differentiated ASCs nor with mature adipocytes. However, we now received the result of acLDL uptake by diffASCs, evident by the simultaneously visible lipid droplets. Further investigations will have to uncover whether this occurrence is conditioned by a starting transdifferentiation of diffASCs or is an independently existing property of these cells.

In the direct co-culture setting, a marked beneficial effect towards the formation of vascular-like structures was observed. It has been shown before that ASCs support the formation of vascular-like structures and their maturation and maintenance through soluble factors like fibroblast growth factors, VEGFs, hepatocyte growth factor or transforming growth factor- $\beta$ and angiopoietin I and II (Zhang et al., 2015; Lin et al., 2016; Xue et al., 2017; Xie et al., 2016; Strassburg et al., 2016). The results obtained from mono- and indirect co-culture confirm the presence of non-differentiated or dedifferentiated ASCs in all conditions and support the hypothesis of a beneficial effect of ASCs on the formation of vascular-like structures. However, the development of vascular-like structures was not detected in the indirect co-culture setting although paracrine signaling would have been possible through 
the shared culture medium. Therefore, angiogenic processes must have been based on a direct cellular interaction, e.g., by functioning as perivascular cells and supporting long-term maintenance of formed structures as described by Rohringer et al. and others (Merfeld-Clauss et al., 2010; Rohringer et al., 2014; Cun et al., 2015). A possible mode of interaction between ECs and perivascular cells via the expression of platelet derived growth factor $\beta$ by ECs and its subsequent binding to the extracellular matrix and by mural cell types has been reported by Gaengel et al. (2009). This condition may additionally serve as explanation for the absent adipocyte function in the indirect co-culture. MvECs might have still supported adipocyte development in direct co-culture. However, they equally triggered the development of supporting perivascular cells of (diff)ASCs which led to a compensation of the effect. Furthermore, as diffASCs were seeded and differentiated two weeks prior to mvECs, they were probably able to synthesize and embed different tissue specific extracellular matrix components like collagen type IV, laminin or fibronectin as described before (Sorrell et al., 2011; Kubo et al., 2000). In fact, the cellular structures spread over several levels in the culture, which became evident through microscopic imaging over hundreds of $\mu \mathrm{m}$. This observation supports the assumption of the development of a 3D-like arrangement of matrix and cellular structures in the culture. Some of these matrix components might not only have facilitated enhanced EC attachment, but also mediated EC migration, sprouting and vessel stabilization during angiogenetic processes as reported elsewhere (Estrach et al., 2011; Kim et al., 2002; Bahramsoltani et al., 2014). These assumptions are supported by beneficial effects on angiogenic processes by decellularized AT, e.g., shown by Sorrell et al. (2011). Additionally, the enhanced binding and presentation of angiogenic factors, derived from the medium or released by diffASCs, might have supported angiogenic processes of ECs (reviewed in Neve et al., 2014). Determination of the components responsible for the enhanced formation of vascular-like structures will be part of further research. To avoid a possible overlap of beneficial influencing by matrix components and cellular signals, solely the influence of decellularized matrix components on angiogenic processes should be evaluated. Independent of the direct or indirect pathway, the developed defined co-culture medium facilitated a sufficient level of crosstalk between the cell types to allow the formation of vascular-like structures and their maintenance and may therefore be rated as appropriate for the set-up of adipocyte/EC co-cultures in terms of engineering vascularized AT. As the 3D arrangement was as well confirmed to support adipogenic differentiation next to angiogenic developments, future studies will have to address a $3 \mathrm{D}$ co-culture in constructs of several $\mathrm{mm}$ in each dimension (Miyamoto et al., 2017). A 3D arrangement will bring the culture even closer to the in vivo situation.

\section{Conclusion and outlook}

With this work we have developed a suitable serum-free, defined adipocyte/EC co-culture medium, which not only keeps diffASCs and mvECs differentiated and functional over time but also allows for sufficient adipocyte/endothelial crosstalk to support the formation of vascular-like structures. Based on this, we managed to build and maintain a vascularized AT model under completely defined medium conditions. Through the replacement of serum while maintaining de novo angiogenesis by the cells, the system thereby represents a very valuable alternative to classically used serum-based approaches. Furthermore, the model promises higher robustness, reproducibility and reliability of the results.

In future studies, the system's ability to represent native human tissue adequately has to be further evaluated to allow for its validation as an in vitro model for various applications. The model may be suitable to replace animal trials for the elucidation of disease mechanisms, the screening for new drugs and the assessment of corresponding safety levels. In combination with existing approaches to engineer epidermis and dermis, the defined adipose tissue model may furthermore be used to generate a full skin equivalent, which is also urgently needed as an in vitro test system. The developed CoM may additionally be suitable for other tissue culture approaches after some cell specific adjustments and thereby may contribute to a general reduction of the use of FBS in cell and tissue culture. Taken together, the application of the defined co-culture medium may reduce animal use both by reduction of the use of FBS in vitro and replacement of animal tests.

\section{References}

Aoki, S., Toda, S., Sakemi, T. and Sugihara, H. (2003). Coculture of endothelial cells and mature adipocytes actively promotes immature preadipocyte development in vitro. Cell Struct Funct 28, 55-60. doi:10.1247/csf.28.55

Arnal-Pastor, M., Martínez-Ramos, C., Vallés-Lluch, A. and Pradas, M. M. (2016). Influence of scaffold morphology on co-cultures of human endothelial and adipose tissue-derived stem cells. J Biomed Mater A 104, 1523-1533. doi:10.1002/ jbm.a.35682

Aubin, K., Vincent, C., Proulx, M. et al. (2015). Creating capillary networks within human engineered tissues: Impact of adipocytes and their secretory products. Acta Biomater 11, 333-345. doi:10.1016/j.actbio.2014.09.044

Bahramsoltani, M., Slosarek, I., Spiegelaere, W. and Plendl, J. (2014). Angiogenesis and collagen type IV expression in different endothelial cell culture systems. Anat Histol Embryol 43, 103-115. doi:10.1111/ahe.12052

Baptista, P. M., Siddiqui, M. M., Lozier, G. et al. (2011). The use of whole organ decellularization for the generation of a vascularized liver organoid. Hepatology 53, 604-617. doi:10.1002/ hep. 24067

Bazzoni, G. and Dejana, E. (2004). Endothelial cell-to-cell junctions: Molecular organization and role in vascular homeostasis. Physiol Rev 84, 869-901. doi:10.1152/physrev.00035.2003

Bellas, E., Marra, K. G. and Kaplan, D. L. (2013). Sustainable three-dimensional tissue model of human adipose tissue. Tissue Eng Part C Methods 19, 745-754. doi:10.1089/ten. tec.2012.0620

Cao, Y. (2007). Angiogenesis modulates adipogenesis and obesity. J Clin Invest 117, 2362-2368. doi:10.1172/JCI32239

Cao, Y. (2010). Adipose tissue angiogenesis as a therapeutic tar- 
get for obesity and metabolic diseases. Nat Rev Drug Discov 9, 107-115. doi:10.1038/nrd3055

Chen, S.-Y., Mahabole, M., Horesh, E. et al. (2014). Isolation and characterization of mesenchymal progenitor cells from human orbital adipose tissue. Invest Ophthalmol Visl Sci 55, 4842-4852. doi:10.1167/iovs.14-14441

Coelho, M., Oliveira, T. and Fernandes, R. (2013). Biochemistry of adipose tissue: An endocrine organ. Arch Med Sci 9, 191200. doi:10.5114/aoms.2013.33181

Cun, X., Xie, J., Lin, S. et al. (2015). Gene profile of soluble growth factors involved in angiogenesis, in an adipose-derived stromal cell/endothelial cell co-culture, 3D gel model. Cell Prolif 48, 405-412. doi:10.1111/cpr.12193

Estrach, S., Cailleteau, L., Franco, C. A. et al. (2011). Laminin-binding integrins induce D114 expression and notch signaling in endothelial cellsnovelty and significance. Circ Res 109, 172-182. doi:10.1161/CIRCRESAHA.111.240622

Fukumura, D., Ushiyama, A., Duda, D. G. et al. (2003). Paracrine regulation of angiogenesis and adipocyte differentiation during in vivo adipogenesis. Circ Res 93, e88-e97. doi:10.1161/01.RES.0000099243.20096.FA

Gaengel, K., Genové, G., Armulik, A. and Betsholtz, C. (2009). Endothelial-mural cell signaling in vascular development and angiogenesis. Arterioscler Thromb Vasc Biol 29, 630-638. doi:10.1161/ATVBAHA.107.161521

Galic, S., Oakhill, J. S. and Steinberg, G. R. (2010). Adipose tissue as an endocrine organ. Mol Cell Endocrinol 316, 129139. doi:10.1016/j.mce.2009.08.018

Gstraunthaler, G. (2003). Alternatives to the use of fetal bovine serum: Serum-free cell culture. ALTEX 20, 275-281. https:// www.altex.org/index.php/altex/article/view/1012

Gstraunthaler, G., Lindl, T. and van der Valk, J. (2013). A plea to reduce or replace fetal bovine serum in cell culture media. Cytotechnology 65, 791-793. doi:10.1007/s10616-013-9633-8

Hartung, T., Balls, M., Bardouille, C. et al. (2002). Good cell culture practice. ECVAM good cell culture practice task force report 1. Altern Lab Anim 30, 407-414.

Huber, B. and Kluger, P. J. (2015). Decelerating mature adipocyte dedifferentiation by media composition. Tissue Eng Part C Methods 21, 1237-1245. doi:10.1089/ten.tec.2015.0166

Huber, B., Borchers, K., Tovar, G. E. and Kluger, P. J. (2015a). Methacrylated gelatin and mature adipocytes are promising components for adipose tissue engineering. J Biomater Appl 30, 699-710. doi:10.1177/0885328215587450

Huber, B., Volz, A. C. and Kluger, P. J. (2015b). Understanding the effects of mature adipocytes and endothelial cells on fatty acid metabolism and vascular tone in physiological fatty tissue for vascularized adipose tissue engineering. Cell Tissue Res 362, 269-279. doi:10.1007/s00441-015-2274-9

Huber, B., Czaja, A. M. and Kluger, P. J. (2016a). Influence of epidermal growth factor (EGF) and hydrocortisone on the co-culture of mature adipocytes and endothelial cells for vascularized adipose tissue engineering. Cell Biol Int 40, 569578. doi:10.1002/cbin.10595

Huber, B., Engelhardt, S., Meyer, W. et al. (2016b). Blood-vessel mimicking structures by stereolithographic fabrication of small porous tubes using cytocompatible polyacrylate elastomers, biofunctionalization and endothelialization. J Funct Biomater 7, 11. doi:10.3390/jfb7020011

Hutley, L. J., Herington, A. C., Shurety, W. et al. (2001). Human adipose tissue endothelial cells promote preadipocyte proliferation. Am J Physiol-Endocrinol Metab 281, E1037-E1044. doi:10.1152/ajpendo.2001.281.5.E1037

Huttala, O., Palmroth, M., Hemminki, P. et al. (2018). Development of versatile human in vitro vascularized adipose tissue model with serum-free angiogenesis and natural adipogenesis induction. Basic Clin Pharmacol Toxicol, Epub ahead of print. doi:10.1111/bcpt.12987

Ikegame, Y., Yamashita, K., Hayashi, S.-I. et al. (2011). Comparison of mesenchymal stem cells from adipose tissue and bone marrow for ischemic stroke therapy. Cytotherapy 13, 675-685. doi:10.3109/14653249.2010.549122

Jacobsson, B. and Smith, U. (1972). Effect of cell size on lipolysis and antilipolytic action of insulin in human fat cells. J Lipid Res 13, 651-656.

Kim, S., Bakre, M., Yin, H. and Varner, J. A. (2002). Inhibition of endothelial cell survival and angiogenesis by protein kinase A. J Clin Invest 110, 933-941. doi:10.1172/JCI14268

Kubo, Y., Kaidzu, S., Nakajima, I. et al. (2000). Organization of extracellular matrix components during differentiation of adipocytes in long-term culture. In Vitro Cell Dev Biol Anim 36, 38-44. doi:10.1290/1071-2690(2000)036<0038:OOEMCD $>2.0 . \mathrm{CO} ; 2$

Lin, S., Xie, J., Gong, T. et al. (2016). Smad signal pathway regulates angiogenesis via endothelial cell in an adipose-derived stromal cell/endothelial cell co-culture, 3D gel model. $\mathrm{Mol}$ Cell Biochem 412, 281-288. doi:10.1007/s11010-015-2634-5

Marcinkiewicz, A., Gauthier, D., Garcia, A. and Brasaemle, D. L. (2006). The phosphorylation of serine 492 of perilipin a directs lipid droplet fragmentation and dispersion. $J$ Biol Chem 281, 11901-11909. doi:10.1074/jbc.M600171200

Merfeld-Clauss, S., Gollahalli, N., March, K. L. and Traktuev, D. O. (2010). Adipose tissue progenitor cells directly interact with endothelial cells to induce vascular network formation. Tissue Eng Part A 16, 2953-2966. doi:10.1089/ten.tea.2009.0635

Miyamoto, Y., Ikeuchi, M., Noguchi, H. et al. (2017). Enhanced adipogenic differentiation of human adipose-derived stem cells in an in vitro microenvironment: The preparation of adipose-like microtissues using a three-dimensional culture. Cell Med 9, 35-44. doi:10.3727/215517916X693096

Moya, M. L., Cheng, M. H., Huang, J. J. et al. (2010). The effect of FGF-1 loaded alginate microbeads on neovascularization and adipogenesis in a vascular pedicle model of adipose tissue engineering. Biomaterials 31, 2816-2826. doi:10.1016/j. biomaterials.2009.12.053

Neve, A., Cantatore, F. P., Maruotti, N. et al. (2014). Extracellular matrix modulates angiogenesis in physiological and pathological conditions. BioMed Res Int 2014, 756078. doi: $10.1155 / 2014 / 756078$

Navina, S. and Singh, V. P. (2015). Relationship between obesity and pancreatitis. Pancreapedia. doi:10.3998/panc.2015.18

Nomi, M., Miyake, H., Sugita, Y. et al. (2006). Role of growth 
factors and endothelial cells in therapeutic angiogenesis and tissue engineering. Curr Stem Cell Res Ther 1, 333-343. doi:10.2174/157488806778226777

Pellegrinelli, V., Rouault, C., Veyrie, N. et al. (2013). Endothelial cells from visceral adipose tissue disrupt adipocyte functions in a 3D setting: Partial rescue by angiopoietin-1. Diabetes 63, 535-549. doi:10.2337/db13-0537

Peyrin-Biroulet, L., Chamaillard, M., Gonzalez, F. et al. (2007). Mesenteric fat in Crohn's disease: A pathogenetic hallmark or an innocent bystander? Gut 56, 577-583. doi:10.1136/ gut.2005.082925

Rajashekhar, G., Roell, W., Traktuev, D. et al. (2008). Adipogenesis of adipose stromal cells is reduced by endothelial cell co-cultivation: Role for Wnt-signaling. FASEB J 22, Suppl.

Rohringer, S., Hofbauer, P., Schneider, K. H. et al. (2014). Mechanisms of vasculogenesis in 3D fibrin matrices mediated by the interaction of adipose-derived stem cells and endothelial cells. Angiogenesis 17, 921-933. doi:10.1007/s10456-0149439-0

Russell, W. M. S. and Burch, R. L. (1959). The principles of humane experimental technique. http://altweb.jhsph.edu/pubs/ books/humane exp/het-toc

Silha, J. V., Krsek, M., Sucharda, P. and Murphy, L. J. (2005). Angiogenic factors are elevated in overweight and obese individuals. Int J Obes (Lond) 29, 1308-1314. doi:10.1038/ sj.ijo.0802987

Sorrell, J. M., Baber, M. A., Traktuev, D. O. et al. (2011). The creation of an in vitro adipose tissue that contains a vascular-adipocyte complex. Biomaterials 32, 9667-9676. doi:10.1016/j. biomaterials.2011.08.090

Strassburg, S., Nienhueser, H., Björn Stark, G. et al. (2016). Co-culture of adipose-derived stem cells and endothelial cells in fibrin induces angiogenesis and vasculogenesis in a chorioallantoic membrane model. J Tissue Eng Reg Med 10, 496-506. doi:10.1002/term. 1769

Tang, W., Zeve, D., Suh, J. M. et al. (2008). White fat progenitor cells reside in the adipose vasculature. Science 322, 583-586. doi:10.1126/science.1156232

van der Valk, J., Mellor, D., Brands, R. et al. (2004). The humane collection of fetal bovine serum and possibilities for serum-free cell and tissue culture. Toxicol In Vitro 18, 1-12. doi:10.1016/j.tiv.2003.08.009

van der Valk, J., Brunner, D., De Smet, K. et al. (2010). Optimization of chemically defined cell culture media - Replacing fetal bovine serum in mammalian in vitro methods. Toxicol In Vitro 24, 1053-1063. doi:10.1016/j.tiv.2010.03.016

Volz, A. C., Huber, B. and Kluger, P. J. (2016). Adipose-derived stem cell differentiation as a basic tool for vascularized adipose tissue engineering. Differentiation 92, 52-64. doi:10.1016/j. diff.2016.02.003
Volz, A.-C., Huber, B., Schwandt, A. M. and Kluger, P. J. (2017). EGF and hydrocortisone as critical factors for the co-culture of adipogenic differentiated ASCs and endothelial cells. Differentiation 95, 21-30. doi:10.1016/j.diff.2017.01.002

Volz, A.-C. and Kluger, P. J. (2018). Completely serum-free and chemically defined adipocyte development and maintenance. Cytotherapy 20, 576-588. doi:10.1016/j.jcyt.2018.01.004

Xie, Q., Xie, J., Zhong, J. et al. (2016). Hypoxia enhances angiogenesis in an adipose-derived stromal cell/endothelial cell co-culture 3D gel model. Cell Prolif 49, 236-245. doi:10.1111/ cpr. 12244

Xu, H., Barnes, G. T., Yang, Q. et al. (2003). Chronic inflammation in fat plays a crucial role in the development of obesity-related insulin resistance. J Clin Invest 112, 1821-1830. doi:10.1172/JCI19451

Xue, C., Xie, J., Zhao, D. et al. (2017). The JAK/STAT3 signalling pathway regulated angiogenesis in an endothelial cell/ adipose-derived stromal cell co-culture, 3D gel model. Cell Prolif 50, e12307. doi:10.1111/cpr.12307

Yao, R., Du, Y., Zhang, R. et al. (2013a). A biomimetic physiological model for human adipose tissue by adipocytes and endothelial cell cocultures with spatially controlled distribution. Biomed Mater 8, 045005. doi:10.1088/1748-6041/8/4/045005

Yao, R., Zhang, R., Lin, F. and Luan, J. (2013b). Biomimetic injectable HUVEC-adipocytes/collagen/alginate microsphere co-cultures for adipose tissue engineering. Biotechnol Bioeng 110, 1430-1443. doi:10.1002/bit.24784

Zhang, Q., Hubenak, J., Iyyanki, T. et al. (2015). Engineering vascularized soft tissue flaps in an animal model using human adipose-derived stem cells and VEGF + PLGA/PEG microspheres on a collagen-chitosan scaffold with a flow-through vascular pedicle. Biomaterials 73, 198-213. doi:10.1016/j. biomaterials.2015.09.024

\section{Conflict of interest}

The authors declare no conflict of interest.

\section{Acknowledgements}

The authors thank Dr Ulrich Ziegler from the Klinik Charlottenhaus in Stuttgart, Germany for the kind provision of human fatty tissue and human skin biopsies from plastic surgery. Additionally, the authors especially thank Ursula Csacsko from Reutlingen University, Germany for technical support in the cell culture work. This work was supported by the Federal Ministry for Education and Research (AdipoDiff; \#03FH012PX4) in the line FHprofUnt. 